\title{
A comparative approach to understanding tissue-specific expression of uncoupling protein 1 expression in adipose
}

\section{tissue}

\section{Andrew Shore ${ }^{1}$, Richard D. Emes ${ }^{2}$, Frank Wessely ${ }^{2}$, Paul Kemp ${ }^{3}$, Clemente Cillo ${ }^{4}$, Maria D'Armiento ${ }^{5}$, Nigel Hoggard' ${ }^{6}$ and Michael A. Lomax ${ }^{7 *}$}

1 School of Biosciences, Cardiff University, Cardiff, UK

${ }^{2}$ School of Veterinary Medicine and Science, University of Nottingham, Sutton Bonington Campus, Leicestershire, UK

${ }^{3}$ Faculty of Medicine, National Heart and Lung Institute, Imperial College London, London, UK

${ }^{4}$ Department of Clinical and Experimental Medicine, Federico II University Medical School, Naples, Italy

${ }^{5}$ Department of Biomorphological and Functional Sciences, Federico II University Medical School, Naples, Italy

${ }^{6}$ Rowett Institute of Nutrition and Health, Aberdeen Centre for Energy Regulation and Obesity, University of Aberdeen, Aberdeen, UK

7 School of Biosciences, University of Nottingham, Sutton Bonington Campus, Leicestershire, UK

\section{Edited by:}

Elvira Larqué, University of Murcia, Spain

Reviewed by:

Michael C. Satterfield, Texas A\&M University, USA

Qiao LI, University of Ottawa, Canada

${ }^{*}$ Correspondence:

Michael A. Lomax, University of Nottingham, School of Biosciences,

Sutton Bonington Campus,

Loughborough, Leicestershire

LE125RD, UK.

e-mail:michael.lomax@

nottingham.ac.uk
The thermoregulatory function of brown adipose tissue (BAT) is due to the tissue-specific expression of uncoupling protein 1 (UCP1) which is thought to have evolved in early mammals. We report that a CpG island close to the UCP1 transcription start site is highly conserved in all 29 vertebrates examined apart from the mouse and xenopus. Using methylation sensitive restriction digest and bisulfite mapping we show that the $\mathrm{CpG}$ island in both the bovine and human is largely un-methylated and is not related to differences in UCP1 expression between white and BAT. Tissue-specific expression of UCP1 has been proposed to be regulated by a conserved $5^{\prime}$ distal enhancer which has been reported to be absent in marsupials. We demonstrate that the enhancer, is also absent in five eutherians as well as marsupials, monotremes, amphibians, and fish, is present in pigs despite UCP1 having become a pseudogene, and that absence of the enhancer element does not relate to BAT-specific UCP1 expression. We identify an additional putative $5^{\prime}$ regulatory unit which is conserved in 14 eutherian species but absent in other eutherians and vertebrates, but again unrelated to UCP1 expression. We conclude that despite clear evidence of conservation of regulatory elements in the UCP1 $5^{\prime}$ untranslated region, this does not appear to be related to species or tissues-specific expression of UCP1.

Keywords: CpG islands, methylation, uncoupling protein 1, phylogenic analysis

\section{INTRODUCTION}

In eutherians, non-shivering thermogenesis (NST) occurs in brown adipose tissue (BAT) which expresses a tissue-specific gene, uncoupling protein 1 (UCP1; Cannon and Nedergaard, 2004). This gene codes for a mitochondrial protein with the ability to uncouple oxidative phosphorylation and generate heat. Recently BAT has been identified in adult humans and has been suggested to offer a potential target to increase energy expenditure and treat obesity(Nedergaard et al., 2007).

The expression of UCP1 is cell-specific to brown adipocytes and has been identified in all mammalian neonates so far examined except the pig, in which exons 3-5 were deleted about 20 million years ago (Berg et al., 2006). BAT-specific UCP1 expression is a feature of small mammals, hibernators, and newborns and is thought to have originated prior to the Eutherian mammal radiation as it has been found in the rock elephant shrew, a member of the Afrotherian mammalian lineage (Mzilikazi et al., 2007). Recent discoveries of UCP1 in non-eutherian marsupials, and of UCP1 orthologs in the non-mammalian vertebrates, frogs, and fish, expressed in liver and muscle, respectively, have questioned this view (Klingenspor et al., 2008; Hughes et al., 2009). Phylogenetic analysis has demonstrated rapid evolution of UCP1 on the Eutherian lineage and suggested that a model of relaxed constraints as predicted from the coevolution of genes which have taken over some of UCP1 function, rather than directional selection, seems to be involved (Hughes et al., 2009). Evidence to support a role of the UCP2 and 3 in oxidative stress suggests that subfunctionalization of these paralogs allowed the divergence of the BAT-specific expression of UCP1 and its role in NST (Klingenspor et al., 2008).

Most newborn mammals are particularly vulnerable to hypothermia, and NST in BAT plays an important role depending on the thermoregulatory behavior of different mammals (Symonds and Lomax, 1992). In altricious newborn such as rodents, pups are born blind and naked, and require the protection of a nest environment to prevent hypothermia until BAT becomes active a few days after birth (Cannon and Nedergaard, 2004). Immature newborns (e.g., hamster) only recruit NST in BAT a week or more after birth with marsupials being an extreme group of immature mammals who do not develop independent NST until the young need to leave the pouch. In contrast to altricious and immature newborns, in precocious mammals (e.g., cows and sheep), BAT develops during fetal life with maximal thermogenic 
activity occurring immediately after birth to allow the newborn to quickly achieve independent thermoregulation (Symonds and Lomax, 1992). Human fetuses and neonates also possess BAT and fit best into the precocial group (Cannon and Nedergaard, 2004) although BAT has been identified in adult humans (McKinnon and Docherty, 2001).

The exact mechanism which confers BAT-specific expression of UCP1 is not known. Studies on the rodent promoter have revealed a highly conserved 221 bp enhancer element located approximately $-2.5 \mathrm{~kb}$ from the transcriptional start that confers both hormonal and tissue-specific responses (Cassard-Doulcier et al., 1998). The enhancer unit is also highly conserved across a $5 \mathrm{~kb}$ genomic sequence upstream of the UCP1 transcription start site in eutherians, including the Afrotherian species but could not be found in marsupials, despite cold-induced UCP1 expression in BAT (Hughes et al., 2009). In a recent study we have proposed that tissue-specific expression may be dictated by the methylation of CpGs in cyclic AMP response elements in the enhancer unit (Shore et al., 2010). Methylation of CpGs in CpG islands (CGI) in the promoter may also confer tissue-specific expression of UCP1 (Kiskinis et al., 2007). Alternatively, tissue-specific expression of UCP1 during development may be governed by the expression of transcriptional regulators as reported in our previous studies (Lomax et al., 2007).

CpGs are generally methylated in the genome except where they occur in CGI around the start of transcription of genes (Sakurai et al., 2006). These CGI, are a feature of TATA-less promoters, and can act as strong promoters of transcription, this effect being modulated by the degree of CpG methylation. Identification of regions of genomic DNA that have been conserved across divergent species is a commonly used method of indicating important regulatory elements.

Here we employ bioinformatic and molecular approaches to demonstrate that despite evidence of conservation of a $\mathrm{CpG}$ island, as well as regulatory elements, in the UCP1 promoter in mammals and vertebrates, these are insufficient to explain expression differences between mammalian species and tissues.

\section{MATERIALS AND METHODS TISSUES}

Bovine perirenal brown fat was obtained from a 1-day-old male calf. Human fetal samples were obtained from legally approved therapeutic terminations at the Department of Pathology University of Naples Federico II under the control of the University's Guidelines for Human Experimentation. Informed consent was obtained from all the subjects involved in the experiments and the study protocols were reviewed and approved by the University Ethical Committee. The age of the fetuses was calculated from anamnesis and ultrasonographic data, to be in the range from 22 to 34 gestational weeks. Tissues were dissected, typically within $2 \mathrm{~h}$ after death. The biopsies of perirenal fetal BAT were immediately frozen in liquid nitrogen and then stored in a freezer at $-80^{\circ} \mathrm{C}$ until DNA/RNA extraction. Human subcutaneous and omental adipose tissue was taken from the abdominal subcutaneous wall, during an operation for vertical banded gastroplasty, from obese female patients. Adipose tissue samples were obtained within $5 \mathrm{~min}$ of the tissue being extracted from the patients and frozen immediately in liquid nitrogen. Subjects had fasted overnight prior to surgery. All patients provided informed written consent before inclusion in the study. The study was approved by the Grampian Research Ethics committee.

\section{Cpg ISLAND PREDICTION}

For each UCP1 ortholog, $5 \mathrm{~kb}$ of genomic DNA upstream of the open reading frame start was screened for CGI using a modified version of the CpGLH program (kindly provided by Angie Hinrichs UCSC). Briefly, each sequence is screened for the presence of CG rich regions which fulfill the CGI criteria of at least $200 \mathrm{bp}$ with a minimum of $50 \% \mathrm{C}+\mathrm{G}$ and where the observed number of CpGs divided by the expected number is greater than 0.6 (Gardiner-Garden and Frommer, 1987). The sensitivity of initial screening parameters was modified to identify all possible CGI whilst maintaining the criteria of Gardiner-Garden and Frommer.

\section{ALIGNMENT OF HOMOLOGOUS PROMOTERS}

Regions of conservation between cow-human and cow-mouse DNA upstream of UCP1 were determined using rVISTA (Loots et al., 2002) using the AVID alignment algorithm (Bray et al., 2003). For details see Table A2 in Appendix.

\section{METHYLATION SENSITIVE RESTRICTION DIGESTION}

Restriction enzyme digests were performed on $1 \mu \mathrm{g}$ of genomic DNA extracted from tissues. Primers (Table A3 in Appendix) were designed to cover short and long fragments of the bovine and human CGI in the UCP1 promoter. In the bovine, two restriction enzymes were chosen recognizing the sequence CCGG, HpaII in which digestion is prevented by methylation, and MspI which is not methylation sensitive and acts to correct for incomplete digestion. Two sets of PCR primers were employed, the first with a product size of $288 \mathrm{bp}$ and containing only one CCGG site and a second with product of $407 \mathrm{bp}$ containing five CCGG sites. In the human, two sets of primers amplifying a short (173 bp; one CCGG) and long (426 bp; eight CCGG) region covering part of the human $\mathrm{CpG}$ island, were employed. For these digests $1 \mu \mathrm{g}$ of genomic DNA was incubated with 10 units of HpaII (Fermentas) in the buffer provided ( $33 \mathrm{mM}$ Tris-acetate, $10 \mathrm{mM} \mathrm{Mg}$-acetate, $66 \mathrm{mM} \mathrm{K}$-acetate, $0.1 \mathrm{mg} / \mathrm{ml} \mathrm{BSA}$ ) in a reaction volume of $50 \mu \mathrm{l}$ for $4 \mathrm{~h}$ at $37^{\circ} \mathrm{C}$ before the enzyme was heat inactivated at $65^{\circ} \mathrm{C}$ for $20 \mathrm{~min}$. One microgram aliquots of genomic DNA were also mock-digested under the same conditions but with nuclease free water added instead of HpaII. A final aliquot was digested using 1 unit of MspI (Fermentas) according to the manufacturer's instructions. The resulting digests were analyzed by quantitative real-time PCR (qRTPCR) using primers for the long and short fragments mentioned above. About $18 \mathrm{~S}$ mRNA was used as a reference gene with primers (Table A3 in Appendix) which amplify a fragment that does not contain a CCGG motif. The human UCP1 enhancer region does not possess the sequence CCGG so TaiI was used which cuts ACGT but is blocked by CpG methylation. Complete digestion was gauged using MnlI which cuts CCTC(N)7.

\section{METHYLATED CYTOSINE MAPPING}

Bisulfite conversion of genomic DNA prepared from tissues was carried out essentially as described by Clark et al. (1994). The modified DNA was purified using a desalting column (Promega Wizard 
DNA Clean-Up system; Promega, Madison, WI, USA) Methylation was quantified by pyrosequencing using Pyro Q-CpG software (Biotage, Charlottesvile, VA, USA) and performed by The Genome Centre, Queen Mary, University of London, Charterhouse Square, London EC1M 6BQ. Primer sequences and descriptions are provided (Table A3 in Appendix), products destined to be pyrosequenced were amplified with $5^{\prime}$-biotin-labeled primers to allow purification before sequencing.

\section{REAL-TIME PCR}

Total RNA was extracted from cultured cells and tissue by use of TRI reagent (Sigma, Poole, UK). Before qRTPCR, samples were treated with RNA-free DNase to remove contaminating genomic or plasmid DNA. Complementary DNA was generated using the cDNA synthesis kit from Qiagen. qRTPCR was performed using Sybr green (Qiagen) according to the manufacturer's instructions in Rotor Gene 3000 (Corbett Research, Cambridge, UK). The sequences of the primers used for qRTPCR are given in Table A3 in Appendix. Expression levels for all genes were normalized to the internal control 18s rRNA using the $\Delta \Delta \mathrm{C}_{\mathrm{t}}$ method (Livak and Schmittgen, 2001).

\section{RESULTS}

\section{IDENTIFICATION OF CpG ISLANDS}

UCP1 homologs from vertebrate species with sufficient genomic data were determined using BLAT at the UCSC genome browser. To ensure that the upstream region of true UCP1 orthologs were compared, the conserved synteny of the UCP1 locus in vertebrates was employed to unequivocally identify the upstream untranslated region of UCP1 in vertebrates. In all species examined the coding region for UCP1 is flanked by TBC1D9 upstream and ELMOD2 downstream (Figure A1 in Appendix). Only those annotated UCP1 genes which were located in the conserved gene triplet TBC1D9-UCP1-ELMOD2 were considered. This resulted in 29 vertebrate UCP1 genes analyzed (see Table A1 in Appendix). A approximately $500 \mathrm{bp}$ sequence with sequence similarity to the human UCP1 enhancer was identified in 20 eutherian mammals but was absent in Marmoset, Pika, Ground Squirrel, Shrew, and Hedgehog (Table 1). The enhancer was also absent from the marsupial Opossum, monotreme Platypus, Xenopus, and Zebrafish. A previous study was similarly unable to identify the enhancer in $10 \mathrm{~Kb}$ upstream UTR of the marsupial M. domestica (Jastroch et al., 2008). The enhancer sequence was within the $-5 \mathrm{~kb}$ of the UTR except for Tenrec in which the enhancer sequence started at $-5.486 \mathrm{~Kb}$ (Table A2 in Appendix).

Using a bioinformatic approach, we identified CGI in the UCP1 promoter of different species, fulfilling the criteria originally described by Gardiner-Garden and Frommer (1987). The results clearly demonstrate the existence of a positionally conserved CpG island in the UCP1 5' UTR in 20 mammalian species (Table 1). By reducing the stringency of the algorithm, an additional five species (rat, shrew, opossum, pika, platypus, and Zebrafish) have identifiable CGI which still fulfill the criteria of Gardiner-Garden and Frommer. From this analysis only two species, Mouse and Xenopus do not have a detectable CGI. The positions of the CGI were within $1 \mathrm{~kb}$ upstream of the UCP1 translational start site (TSS) except for the European Hedgehog in which the CpG island was located downstream of the TSS.

\section{Cpg METHYLATION OF THE BOVINE AND HUMAN UCP1 Cpg ISLAND}

The high conservation of the CPG island in the proximal UCP1 promoter across evolutionary time in vertebrates suggests that this region may be of regulatory importance. We therefore next examined the methylation state of the proximal promoter in human and the bovine tissues, in order to establish whether BAT-specific expression of UCP1 is dictated by CpG methylation state of the UCP1 promoter. UCP1 mRNA expression in bovine white adipose tissue (WAT, subcutaneous), BAT (perirenal), and liver were determined by qRTPCR. BAT had significantly greater (200-fold) UCP1 expression than WAT or liver (Figure 1A; $p<0.001$ ). The high expression of UCP1 in BAT was not unexpected since these samples were taken shortly after birth $(8 \mathrm{~h})$ and previous studies, including our own in ruminants, have shown that UCP1 expression is at its highest around parturition in response to the cold extrauterine environment (Symonds and Lomax, 1992). Previous studies have demonstrated that UCP1 expression is high in human fetal BAT (Gavrilova et al., 1988).

Methylation sensitive restriction digests were carried out on genomic DNA extracted from neonatal bovine BAT, subcutaneous WAT, and liver, fetal human BAT, and adult human WAT, (omental and subcutaneous) to determine differences in methylation state between the tissues. Methylation of the bovine proximal promoter CpG island was low in all tissues with a 407 bp product being less than $2 \%$ methylated and a 288 bp product less than $12 \%$. (Figure 1B). There was no significant difference in methylation state of the $\mathrm{CpG}$ island between bovine tissues. It was expected that the $407 \mathrm{bp}$ fragment would be more susceptible to methylation sensitive digestion as this contained more restriction sites, increasing the probability that a methylated site would be encountered by the enzyme. In the human proximal promoter $\mathrm{CpG}$ island, methylation state of fetal BAT was also low $(<14 \%)$ but was significantly $(p<0.05)$ higher (173 bp product, 14\%: 426 bp product, $4 \%$ methylated) than WAT from both depots which were unmethylated (Figure 1C). A similar methylation sensitive restriction digestion approach (see Materials and Methods) demonstrated that the methylation state of a region of the human enhancer was much higher (55-60\%) than the proximal promoter CpG island (Figure 1D). The primers amplified a region that contains this sequence which also lies at the consensus CRE homologous to CRE3 in the mouse.

We next employed bisulfite mapping in order to confirm the apparent low levels of methylation in the bovine CpG island, in the bovine tissues. CGI are difficult to analyze using PCR bisulfite mapping due to the problem of designing primers and although we attempted to amplify $44 \mathrm{CpGs}$ in and around the bovine $\mathrm{CpG}$ island we were only able to produce reliable results for $12 \mathrm{CpGs}$. In agreement with the methylation sensitive restriction digests, all of these CpGs had methylation levels less than 20\% with the majority below $10 \%$ with no significant differences between the tissue types (Figure 1E). For comparison, the values for CpG methylation of the mouse enhancer around CRE3 determined by bisulfite mapping in our previous studies (Shore et al., 2010) have been included in Figure 1E to emphasize the relatively low methylation 


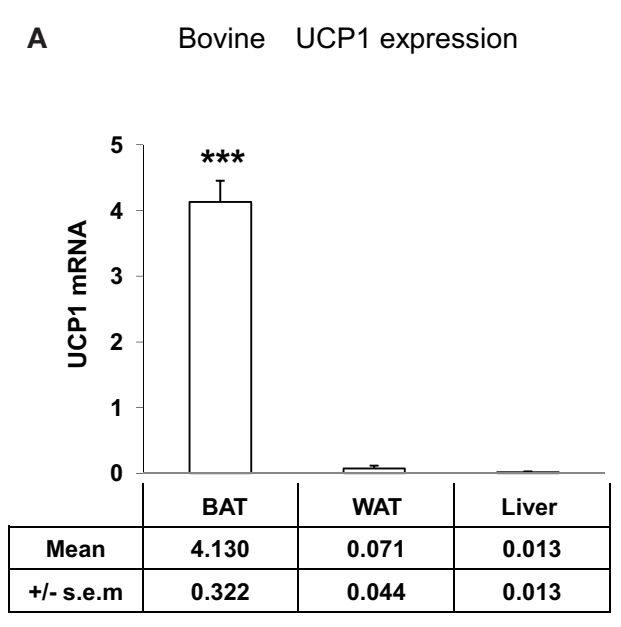

C Human $\mathrm{CpG}$ Island

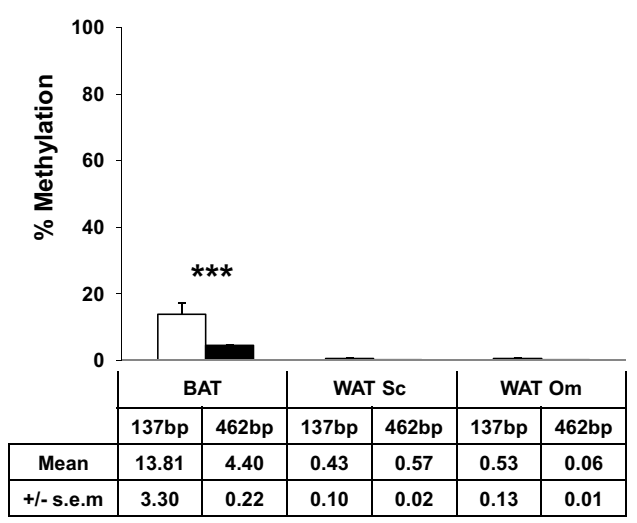

B Bovine $\mathrm{CpG}$ Island

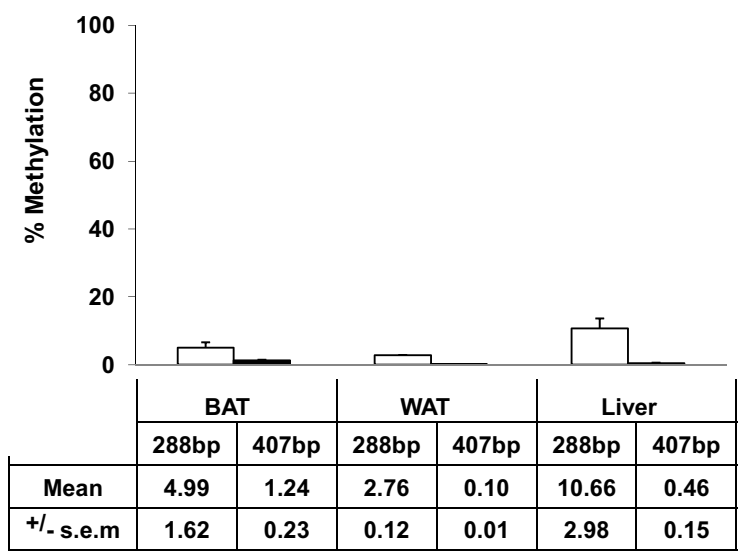

D

Human Enhancer

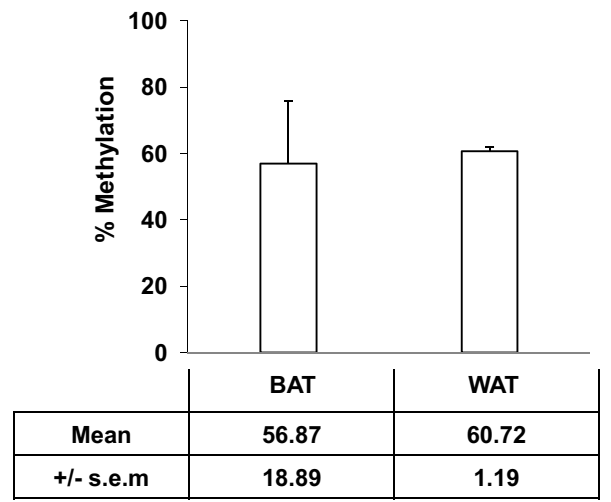

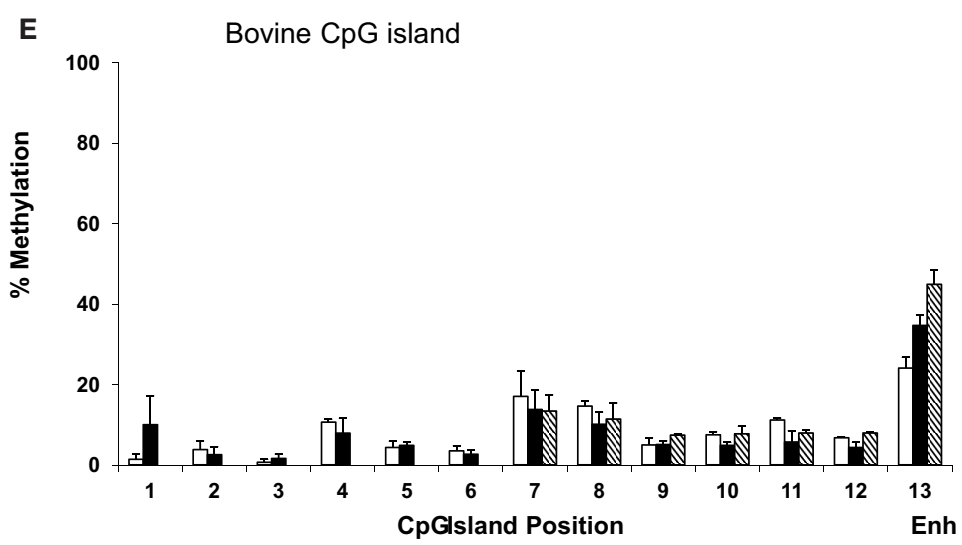

FIGURE 1 | Expression and UCP1 promoter percentage CpG methylation. (A) bovine UCP1 mRNA expression by qRTPCR. Methylation sensitive restriction digest determination of (B) bovine, (C) human $\mathrm{CpG}$ islands, (D) human enhancer, and (E) bisulfite mapping determination of the percentage methylation of $12 \mathrm{CpGs}$ within the bovine $\mathrm{CpG}$ island, in adipose tissues and liver. UCP1 mRNA (A) is expressed relative to ribosomal 18S mRNA. The data are presented as a percentage methylation compared to each respective mock methylated sample for the (B) bovine 288 bp ( $\square$ ) and the 407 bp ( $\square$ ) products and (C) human $173 \mathrm{bp}(\square)$ and the $426 \mathrm{bp}(\boldsymbol{\square})$ products and (D) human enhancer (see Materials and Methods). The amount of UCP1 promoter DNA was quantified by qPCR relative to ribosomal 18S DNA. (E) $\mathrm{CpG}$ dinucleotide methylation in the $U_{c p} 1$ proximal promoter in newborn bovine brown ( $\square$ ) and subcutaneous white adipose tissue ( $\square$ ), and liver (Z). For comparison, values for the mouse enhancer (ENH) BAT, WAT, and liver are presented. DNA was extracted, bisulfite modified, amplified by PCR, and pyrosequenced to determine $\mathrm{CpG}$ methylation over positions 1-12 of the Ucp1 promoter (see Materials and Methods). Missing liver values are due to failed analyses. Values are means \pm SEM from at least three replicates except for (D) which represents the average of duplicates $\pm S D * * *$ BAT significantly greater than other tissues $(p<0.001)$. 
Table 1 | Occurrence and position of CpG island, enhancer and putative regulatory region in relation to the start of UCP1 transcription in 27 vertebrate species.

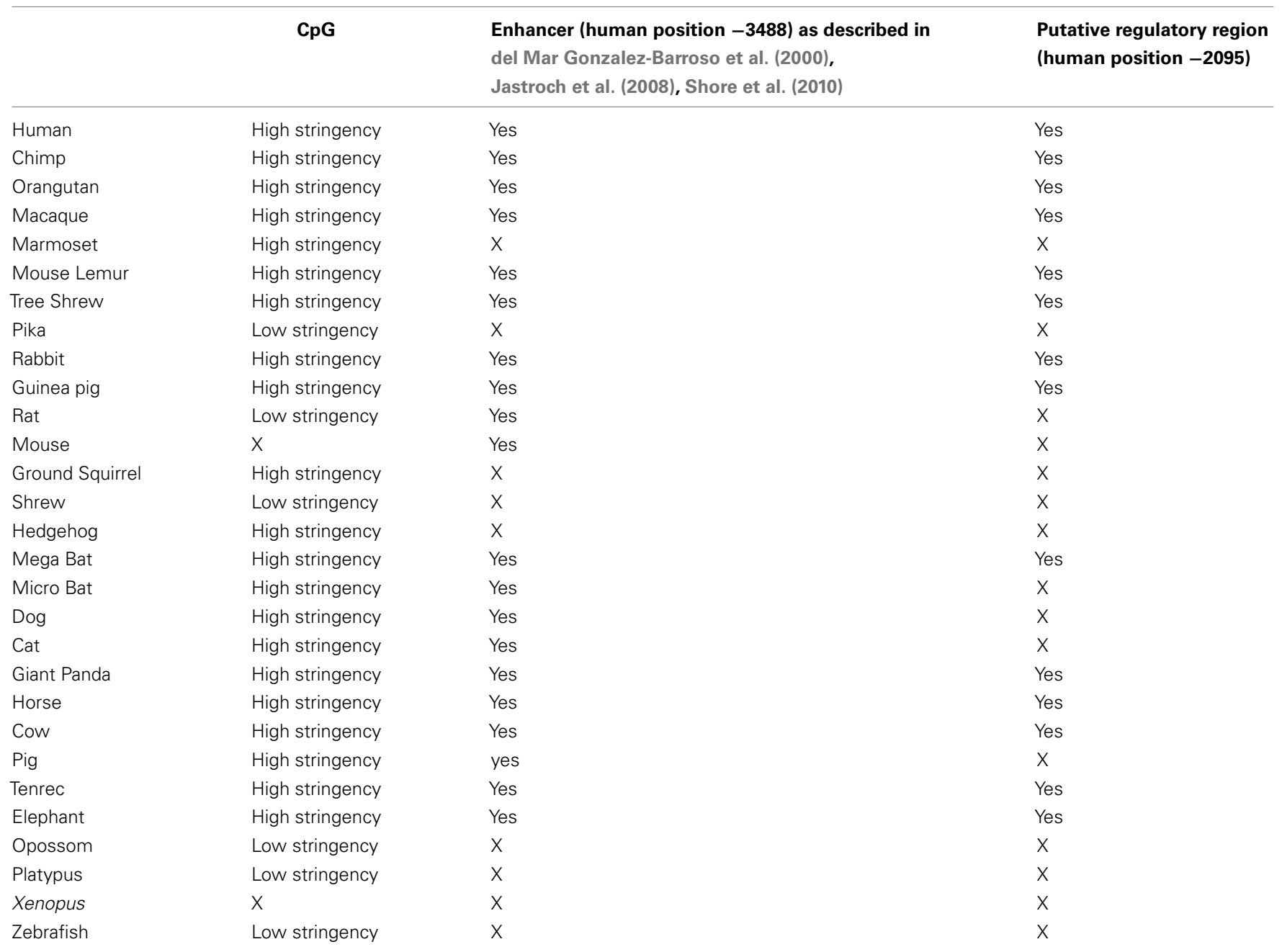

High stringency regions represent $C p G$ islands identified by the $C p G L H$ algorithm with default settings, low stringency regions represent $C p G$ islands identified by the CpGLH algorithm with relaxed settings (see Materials and Methods). Putative Regulatory Region represents a 500 bp region conserved in some species containing multiple consensus response elements.

state of the bovine CpG island. There was insufficient human BAT to carry out a similar bisulfite mapping analysis.

\section{THE POSITION OF A CONSERVED 5' UPSTREAM ENHANCER REGION AND A PUTATIVE REGULATORY REGION IN THE PROMOTER OF UCP1 IN VERTEBRATES}

Since methylation CpG state of the UCP1 promoter was unable to explain brown adipose-specific expression, we next turned our attention to the bioinformatics analysis of the promoter region. Conservation of a $320 \mathrm{bp}$ enhancer in a $10 \mathrm{~Kb}$ region upstream of the UCP1 TSS has been previously reported in eutherians, including the Afrotherian species but not in the marsupial $M$. domestica, (Jastroch et al., 2008). We extended this study to include non-mammalian vertebrates (Table 1). Surprisingly, although we could detect the enhancer box in the $10 \mathrm{~kb}$ sequence upstream of the TSS in 20 eutherian species, it was not present in five eutherians (Marmoset, Pika, Ground Squirrel, Shrew, Hedgehog) despite BAT-specific UCP1 expression in these species. The low coverage (approximately $2 \times$ ) of four of these (Pika, Ground Squirrel, Shrew and Hedgehog) is likely to be insufficient to confidently conclude the lack of this enhancer. However Marmoset has increased coverage $(6 \times)$ and provides greater confidence of the loss of enhancer in mammalian species. Within the marmoset genome the nearest gap upstream of the UCP1 gene is estimated to be $54,083 \mathrm{bp}$ upstream, suggesting that the lack of predicted enhancer is not due to missing sequence data. As expected the enhancer box was not detected in the marsupial Opossum, the monotreme, Platypus, or non-mammalian vertebrates (Xenopus, Zebrafish). Within the mammalian species possessing a $5^{\prime}$ distal enhancer there was remarkable conservation of response element sequences that have been shown to regulate UCP1 transcription in rodent studies, as previously noted by Jastroch et al. (2008; Figures A3-A5 in Appendix). The enhancer sequence was within the $-5 \mathrm{~kb}$ of the UTR except for Tenrec in which the enhancer 
sequence started at $-5.486 \mathrm{~Kb}$ (Table A2 in Appendix). The presence of a conserved enhancer sequence upstream of pig UCP1 is possibly unexpected. The UCP1 gene was predicted to have become a pseudogene approximately 20 million years ago (Berg et al., 2006). If the sole role of the enhancer is associated with UCP1 expression, it would be predicted that following pseudogenization that purifying selection of UCP1 enhancer would be relaxed, resulting in degeneration of conservation by accumulation of mutations. However, the pig enhancer remains well conserved. Pairwise percent identify of Human-cow enhancer is $78.5 \%$ and is only slightly lower in Human-pig (75.9\%). This suggests a possible additional role for the enhancer in pig or that the expression of a truncated form of UCP1 is transcribed in pig.

A second conserved putative regulatory region of approximately 500 bp was noted (Human -2095; usually placed 2200$2700 \mathrm{bp}$ upstream of the TSS in most species) which although present in 14 of the eutherian species, was absent in the nine vertebrate species that we could not find the enhancer, with the exception of rodents (Table 1; Table A2 in Appendix; Figures A3 and A4 in Appendix).

Pairwise comparison of bovine-mouse, or bovine-human promoters using Rvista (Loots et al., 2002) highlighted this conserved putative regulatory region between the human and bovine approximately $2.5 \mathrm{~Kb}$ upstream, but not between bovine and mouse (Figure 2). As expected, a highly conserved peak is visible at approximately $-3.6 \mathrm{~Kb}$ within the conserved enhancer region and contained the conserved transcription factor binding sites previously mentioned above. A second conserved region approximately -1.1 to $-1.6 \mathrm{~kb}$ is conserved between bovine and human but is missing in mouse and rat genomes. The putative regulatory region also contained a number of conserved transcription factor binding sites (CEBP, CREB, DR1, DR3, DR4, PPAR) suggesting the presence of control elements that may be important in regulating species-specific UCP1 expression.

\section{DISCUSSION}

The recent discovery of BAT in adult humans has excited interest in combating obesity by stimulating the expression and activity of UCP1 in brown adipocytes in order to increase energy expenditure. In order to manipulate energy expenditure it is necessary to understand the precise transcriptional regulation of UCP1 and although there have been recent advances in the transcriptional factors and co-regulators required for activating the brown adipogenic gene expression, the mechanisms responsible

Human Chr4
Chimp Chr4
Orangutan Chr4
Macaque Chr4
Marmoset Chr3
Mouse Lemur scaffold 1087
Tree shrew scaffold 4806
Pika scaffold 3061
Rabbit Chr15
Guinea pig scaffold 7
Rat Chr19
Mouse Chr8
Ground squirrel scaffold 3452
Shrew scaffold 1671
Hedgehog scaffold 252452
Mega bat scaffold 2289
Micro bat scaffold 5996
Dog Chr19
Cat ChrB1
Giant Panda GL193536.1
Horse Chr2
Cow Chr17
Pig Chr8
Tenrec scaffold 7417
Elephant scaffold 14
Opossom Chr5
Platypus Ultra33
Xenopus Chr16
Zebrafish Chr1

Human Chr4

Orangutan $\mathrm{Chr}$

Macaque Chr4

Tree shrew scaffold 4806

Guinea pig scaffold 7

Ground squirrel scaffold 345

Shrew scaffold 1671

Mega bat scaffold 2289

Micro bat scaffold 5996

Dog Chr19

Horse Chr2

Cow $\mathrm{Chr} 17$

Tenrec scaffold 7417

Elephant scaffold 14

Opossom Chr5

Platypus Ultra33

Zebrafish Chr1

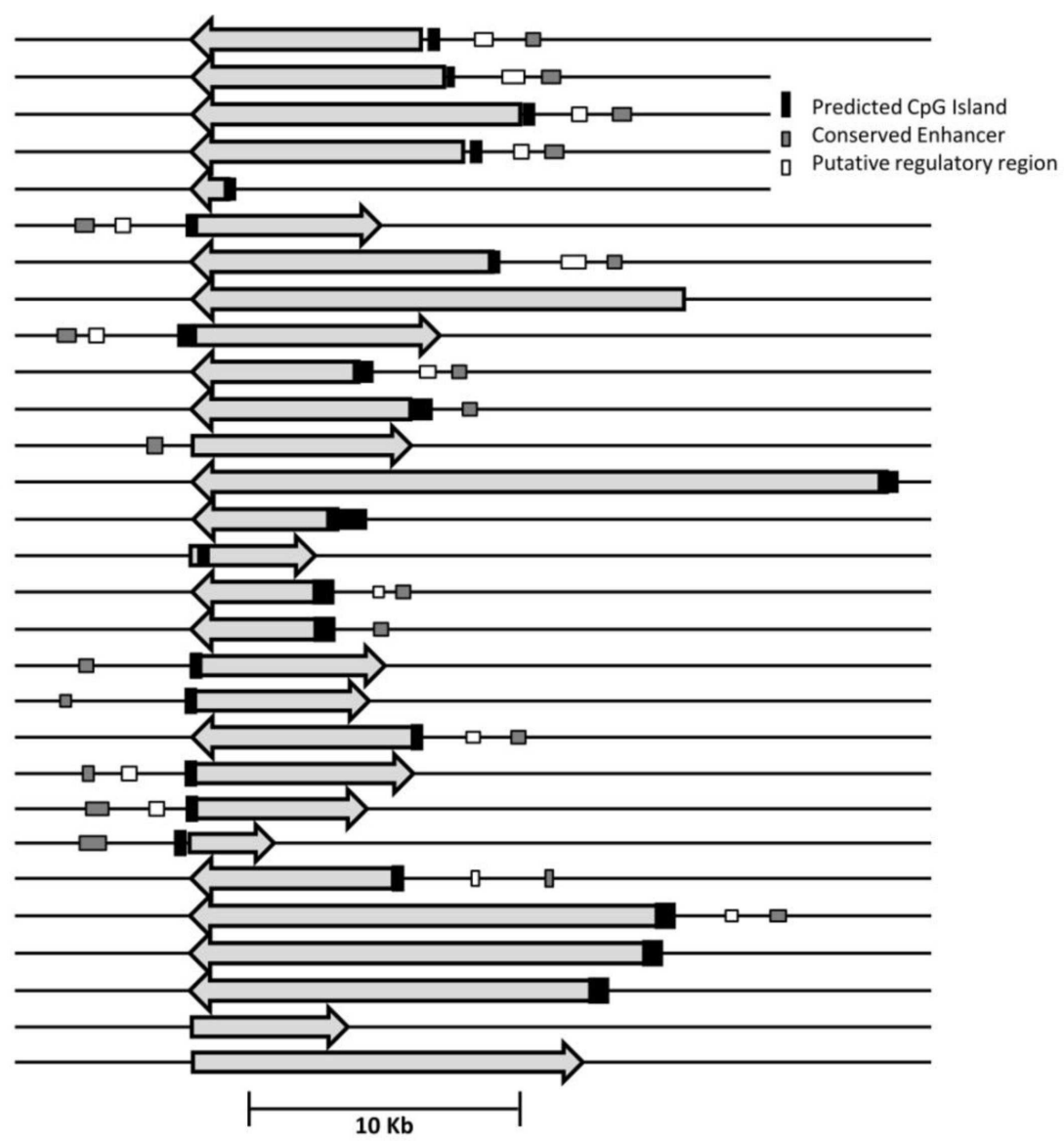

FIGURE 2 | Map of the relative positions of the conserved enhancer, putative regulatory region and predicted $\mathrm{CpG}$ island in the UCP1 promoter of 29 species. All genes are shown in $5^{\prime}-3^{\prime}$ orientation. Arrow represent the region of the UCP1 coding sequence. Differences in arrow length are likely to reflect relative differences in intron sizes. 
for the species-specific and tissue-specific expression of UCP1 are unknown. The vast majority of studies have been carried out in rodents which retain neonatal brown depots into adulthood. In humans neonates, significant amounts of BAT are found in the perirenal and axillary depots, disappearing in adults but being replaced by the recently discovered supraclavicular depots. We and others have reported a similar developmental disappearance of BAT from the perirenal depot in ruminants (Lomax et al., 2007). We have proposed that tissue-specific expression may be dictated by the methylation of specific CpGs in cyclic AMP response elements in the UCP1 enhancer unit (Shore et al., 2010). An alternative suggestion is that methylation of CpGs in CGI in the promoter may confer tissue-specific expression of UCP1 (Kiskinis et al., 2007).

Using a bioinformatic approach we were able to identify a CpG island conserved across 26 of 28 mammalian including marsupials and monotremes (Figure 2). Additionally a CpG island can be identified upstream of the Zebrafish UCP1 transcription start site suggesting a more ancient origin and that this $\mathrm{CpG}$ island predates the divergence of mammals. In the context of the evolution of the CGI in the UCP1 promoter, it is therefore unlikely that the retention of the $\mathrm{CpG}$ island is related to the acquisition of BAT-specific expression since this is a feature only of mammals. This conclusion was supported by our study using methyl sensitive restriction digestion and $\mathrm{qPCR}$ which demonstrates that the methylation state of the bovine $\mathrm{CPG}$ island does not appear to account for the differential expression of UCP1 shown by qPCR between BAT and WAT and that the $\mathrm{CpG}$ island remains essentially demethylated in BAT, WAT, and liver tissues regardless of the level UCP1 expression. These low methylation states were confirmed by pyrosequencing analysis of the region. Though it is possible that some of the unsequenced CpGs show differential methylation levels, we show that there is not a wide ranging difference in methylation state compared with differences in UCP1 expression. These findings were confirmed in the human tissues where there were also low levels of methylation and no apparent difference between fetal BAT and adult WAT promoter methylation despite well documented difference in UCP1 expression between these tissues (Lean and James, 1986).

We have previously observed in mice that $\mathrm{CpG}$ dinucleotide methylation of the Ucp1 distal enhancer exhibits tissue-specific patterns in murine tissue and cell lines and suggested that adipose tissue-specific Ucp1 expression involves demethylation of CpG dinucleotides found in regulatory CREs in the Ucp 1 enhancer, as well as modification of histone tails (Shore et al., 2010). The control of UCP1 expression by a complex series of response elements in the $5^{\prime}$ distal enhancer has been studied in the rodent and human promoter (del Mar Gonzalez-Barroso et al., 2000; Rim and Kozak, 2002) where this enhancer is necessary for both response to drugs and tissue-specific expression. However the observation that marsupial M. domestica expresses UCP1 in response to beta adrenergic stimulation despite there being no identifiable enhancer suggests that other regulatory mechanisms exist (Jastroch et al., 2008). We confirmed this observation and have demonstrated that the enhancer is also absent from the other species Marmoset, Pika, Ground Squirrel, Shrew, and Hedgehog despite evidence that of
BAT-specific expression of UCP1 in these species (Rothwell and Stock, 1985; Loncar, 1990; Liu et al., 1998; Suzuki et al., 2006; Kitao et al., 2007). All of the nine species lacking an identifiable enhancer also lacked the putative regulatory region but further studies are necessary to characterize this region (Figure 2). Taken together the results do not support a role for either $\mathrm{CpG}$ island methylation or the presence of an enhancer unit, in tissue-specific regulation of UCP1 expression.

Our previous study suggested that the loss of adrenergic stimulation of UCP1 expression in perirenal adipose tissue from newborn ruminants is associated with a decrease in the expression of the PPAR $\gamma$ coactivator PGC1 $\alpha$ (Lomax et al., 2007) suggesting that the transcriptional machinery in ruminants may fail to activate the enhancer after birth. In rodents cAMP response elements are present in both the enhancer and the proximal promoter (Rim and Kozak, 2002). We have previously demonstrated using mouse cell lines, that the exact combination of transcription factors binding to cAMP response elements, governs the brown adipocyte-specific expression of PGC1 $\alpha$ and UCP1, in response to cAMP stimulation (Karamanlidis et al., 2007; Karamitri et al., 2009). Further studies in rodents have also suggested synergistic relationships between the transcriptional factors, PPAR $\gamma, \operatorname{PPAR} \alpha$, and PGC1 $\alpha$ in brown adipogenesis (Rim et al., 2004; Xue et al., 2005). Therefore, the species differences in the presence of an enhancer and the patterns of brown fat thermogenesis may depend on the specific combinations and trans-activational prowess of transcription factors, rather than the exact structure of $5^{\prime}$ upstream elements. Further studies are required to identify the role of transcription factors activating the CREB and PPAR response elements identified in the bovine PRR (Figure 2; Figure A2 in Appendix) in the regulation of thermogenesis in different species.

\section{CONCLUSION}

The results presented here demonstrate that mammals possess a highly conserved $\mathrm{CpG}$ island close to the transcription start site on the UCP1 promoter but that methylation of the CpG island does not appear to account for tissue-specific expression of UCP1 in these species. The evolution of the enhancer element appears to be separate from the thermoregulatory function of BAT with species lacking an enhancer being able to increase UCP1 expression in response to cold stimulus, or as in the pig, retain the enhancer despite UCP1 becoming a pseudogene. Therefore, although previous studies in rodents have proposed that regulation of UCP1 expression is mainly targeted at response elements in a complex enhancer, a comparative approach suggests that despite clear evidence of conservation of regulatory elements in the UCP1 $5^{\prime}$ untranslated region, this does not appear to be related to speciesor tissues-specific expression of UCP1. This suggests that the control of mammalian thermogenesis in BAT is not simply due to the evolution of UCP1 promoter elements but the result of a complex interplay between transcriptional regulators and response elements on the UCP1 promoter.

\section{ACKNOWLEDGMENTS}

The work was supported by the BBSRC and the University of Nottingham. 


\section{REFERENCES}

Berg, F., Gustafson, U., and Andersson, L. (2006). The uncoupling protein 1 gene (UCP1) is disrupted in the pig lineage: a genetic explanation for poor thermoregulation in piglets. PLoS Genet. 2:e129. doi:10.1371/journal.pgen.0020129

Bray, N., Dubchak, I., and Pachter, L. (2003). AVID: a global alignment program. Genome Res. 13, 97-102.

Cannon, B., and Nedergaard, J. (2004). Brown adipose tissue: function and physiological significance. Physiol. Rev. 84, 277-359.

Cassard-Doulcier, A. M., Gelly, C., Bouillaud, F., and Ricquier, D. (1998). A 211-bp enhancer of the rat uncoupling protein-1 (UCP-1) gene controls specific and regulated expression in brown adipose tissue. Biochem. J. 333(Pt 2), 243-246.

Clark, S. J., Harrison, J., Paul, C. L., and Frommer, M. (1994). High sensitivity mapping of methylated cytosines. Nucleic Acids Res. 22, 2990-2997.

del Mar Gonzalez-Barroso, M., Pecqueur, C., Gelly, C., Sanchis, D., Alves-Guerra, M. C., Bouillaud, F., et al. (2000). Transcriptional activation of the human ucpl gene in a rodent cell line. Synergism of retinoids, isoproterenol, and thiazolidinedione is mediated by a multipartite response element. J. Biol. Chem. 275, 31722-31732.

Gardiner-Garden, M., and Frommer, M. (1987). CpG islands in vertebrate genomes. J. Mol. Biol. 196, 261-282.

Gavrilova, O. F., Mironov, A. S., Nikolaevskaia, E. E., Rodionov, O. A., and Khurges, E. M. (1988). Genetic mapping of the ilv7434 mutation providing threonine deaminase resistance to isoleucine inhibition in Escherichia coli. Genetika 24, 13-22.

Hughes, D. A., Jastroch, M., Stoneking, M., and Klingenspor, M. (2009). Molecular evolution of UCP1 and the evolutionary history of mammalian non-shivering thermogenesis. BMC Evol. Biol. 9:4. doi:10.1186/1471-2148-9-4

Jastroch, M., Withers, K. W., Taudien, S., Frappell, P. B., Helwig, M., Fromme, T., et al. (2008). Marsupial uncoupling protein 1 sheds light on the evolution of mammalian non-shivering thermogenesis. Physiol. Genomics 32, 161-169.
Karamanlidis, G., Karamitri, A., Docherty, K., Hazlerigg, D. G., and Lomax, M. A. (2007). $\mathrm{C} /$ EBPbeta reprograms white 3T3-L1 preadipocytes to a Brown adipocyte pattern of gene expression. J. Biol. Chem. 282, 24660-24669.

Karamitri, A., Shore, A. M., Docherty, K., Speakman, J. R., and Lomax, M. A. (2009). Combinatorial transcription factor regulation of the cyclic AMP-response element on the Pgclalpha promoter in white 3T3-L1 and brown HIB-1B preadipocytes. J. Biol. Chem. 284, 20738-20752.

Kiskinis, E., Hallberg, M., Christian, M., Olofsson, M., Dilworth, S. M., White, R., et al. (2007). RIP140 directs histone and DNA methylation to silence Ucpl expression in white adipocytes. EMBO J. 26, 4831-4840.

Kitao, N., Yahata, T., Matsumoto, T., Okamatsu-Ogura, Y., Omachi, A., Kimura, K., et al. (2007). Molecular cloning and tissue distribution of uncoupling protein 1 (UCP1) in plateau pika (Ochotona dauurica). J. Vet. Med. Sci. 69, 1065-1068.

Klingenspor, M., Fromme, T., Hughes, D. A. Jr., Manzke, L., Polymeropoulos, E., Riemann, T., et al., (2008). An ancient look at UCP1. Biochim. Biophys. Acta 1777, 637-641.

Lean, M. E. J., and James, W. P. T. (1986). "Brown adipose tissue in man," in Brown Adipose Tissue, eds P. Trayhurn and D. G. Nicholls (London: Edward Arnold), 339-365.

Liu, X. T., Lin, Q. S., Li, Q. F., Huang, C. X., and Sun, R. Y. (1998). Uncoupling protein mRNA, mitochondrial GTP-binding, and T4 5'-deiodinase activity of brown adipose tissue in Daurian ground squirrel during hibernation and arousal. Comp. Biochem. Physiol. Part A Mol. Integr. Physiol. 120, 745-752.

Livak, K. J., and Schmittgen, T. D. (2001). Analysis of relative gene expression data using real-time quantitative PCR and the 2(-Delta Delta C(T)) method. Methods 25, 402-408.

Lomax, M. A., Sadiq, F., Karamanlidis, G., Karamitri, A., Trayhurn, P., and Hazlerigg, D. G. (2007). Ontogenic loss of brown adipose tissue sensitivity to beta-adrenergic stimulation in the ovine. Endocrinology 148, 461-468.

Loncar, D. (1990). Immunoelectron microscopical studies on synthesis and localization of uncoupling protein in brown adipocytes: evidence for cotranslational transport of uncoupling protein into mitochondria. J. Struct. Biol. 105, 133-145.

Loots, G. G., Ovcharenko, I., Pachter, L., Dubchak, I., and Rubin, E. M. (2002). rVista for comparative sequence-based discovery of functional transcription factor binding sites. Genome Res. 12, 832-839.

McKinnon, C. M., and Docherty, K. (2001). Pancreatic duodenal homeobox-1, PDX-1, a major regulator of beta cell identity and function. Diabetologia 44, 1203-1214.

Mzilikazi, N., Jastroch, M., Meyer, C. W., and Klingenspor, M. (2007). The molecular and biochemical basis of non-shivering thermogenesis in an African endemic mammal, Elephantulus myurus. Am. J. Physiol. Regul. Integr. Comp. Physiol. 293, R2120 R2127.

Nedergaard, J., Bengtsson, T., and Cannon, B. (2007). Unexpected evidence for active brown adipose tissue in adult humans. Am. J. Physiol. Endocrinol. Metab. 293, E444-E452.

Rim, J. S., and Kozak, L. P. (2002). Regulatory motifs for CREB-binding protein and $\mathrm{Nfe} 2 \mathrm{l} 2$ transcription factors in the upstream enhancer of the mitochondrial uncoupling protein 1 gene. J. Biol. Chem. 277, 34589-34600.

Rim, J. S., Xue, B., Gawronska-Kozak, B., and Kozak, L. P. (2004). Sequestration of thermogenic transcription factors in the cytoplasm during development of brown adipose tissue. J. Biol. Chem. 279, 25916-25926.

Rothwell, N. J., and Stock, M. J. (1985). Thermogenic capacity and brown adipose tissue activity in the common marmoset. Comp. Biochem. Physiol. A Comp. Physiol. 81, 683-686.

Sakurai, H., Era, T., Jakt, L. M., Okada, M., Nakai, S., and Nishikawa, S. (2006). In vitro modeling of paraxial and lateral mesoderm differentiation reveals early reversibility. Stem Cells 24, 575-586.
Shore, A., Karamitri, A., Kemp, P., Speakman, J. R., and Lomax, M. A. (2010). Role of Ucp1 enhancer methylation and chromatin remodelling in the control of Ucp1 expression in murine adipose tissue. Diabetologia 53, 1164-1173.

Suzuki, D., Murata, Y., and Oda, S. (2006). Cloning of putative uncoupling protein $1 \mathrm{cDNA}$ in a coldintolerant mammal, the house musk shrew (Suncus murinus). Zool. Sci. 23, 1009-1015.

Symonds, M. E., and Lomax, M. A. (1992). Maternal and environmental influences on thermoregulation in the neonate. Proc. Nutr. Soc. 51, 165-172.

Xue, B., Coulter, A., Rim, J. S., Koza, R. A., and Kozak, L. P. (2005). Transcriptional synergy and the regulation of Ucpl during brown adipocyte induction in white fat depots. Mol. Cell. Biol. 25, 8311-8322.

Conflict of Interest Statement: The authors declare that the research was conducted in the absence of any commercial or financial relationships that could be construed as a potential conflict of interest.

Received: 03 October 2012; paper pending published: 02 November 2012; accepted: 10 December 2012; published online: 03 January 2013.

Citation: Shore A, Emes RD, Wessely $F$, Kemp $P$, Cillo $C$, D'Armiento $M$, Hoggard $N$ and Lomax MA (2013) A comparative approach to understanding tissue-specific expression of uncoupling protein 1 expression in adipose tissue. Front. Gene. 3:304. doi: 10.3389/fgene.2012.00304

This article was submitted to Frontiers in Epigenomics and Epigenetics, a specialty of Frontiers in Genetics.

Copyright (c) 2013 Shore, Emes, Wessely, Kemp, Cillo, D'Armiento, Hoggard and Lomax. This is an open-access article distributed under the terms of the Creative Commons Attribution License, which permits use, distribution and reproduction in other forums, provided the original authors and source are credited and subject to any copyright notices concerning any third-party graphics etc. 


\section{APPENDIX}

Table A1 | Genome builds of species investigate.

\begin{tabular}{|c|c|c|}
\hline Common name & Latin name & Genome build \\
\hline Human & Homo sapiens & March 2006 hg18 \\
\hline Chimp & Pan troglodytes & March 2006 panTro2 \\
\hline Orangutan & Pongo pygmaeus abelii & July 2007 ponAbe2 \\
\hline Rhesus & Macaca mulatta & January 2006 rheMac2 \\
\hline Marmoset & Callithrix jacchus & June 2007 calJac1 \\
\hline Mouse lemur & Microcebus murinus & June 2003 micMur1 \\
\hline TreeShrew & Tupaia belangeri & December 2006 tupBel1 \\
\hline Pika & Ochotona princeps & July 2008 ochPri2 \\
\hline Rabbit & Oryctolagus cuniculus & May 2005 oryCun1 \\
\hline Guinea pig & Cavia porcellus & February 2008 cavPor3 \\
\hline Rat & Rattus norvegicus & November 2004 rn4 \\
\hline Mouse & Mus musculus & July 2007 mm9 \\
\hline Ground squirrel & Spermophilus tridecemlineatus & February 2008 speTri1 \\
\hline Shrew & Sorex araneus & June 2006 sorAra1 \\
\hline Hedgehog & Erinaceus europaeus & June 2006 eriEur1 \\
\hline Megabat & Pteropus vampyrus & July 2008 pteVam1 \\
\hline Microbat & Myotis lucifugus & March 2006 myoLuc1 \\
\hline Dog & Canis lupus familiaris & May 2005 canFam2 \\
\hline Cat & Felis catus & March 2006 felCat3 \\
\hline Giant panda & Ailuropoda melanoleuca & AilMel 1.0 December 2009 \\
\hline horse & Equus caballus & September 2007 equCab2 \\
\hline Cow & Bos taurus & November 2009 bosTau6 \\
\hline Pig & Sus scrofa & SGSC Sscrofa9.2 \\
\hline Tenrec & Echinops telfairi & July 2005 echTel1 \\
\hline Elephant & Loxodonta africana & July 2008 loxAfr2 \\
\hline Opossum & Monodelphis domestica & January 2006 monDom4 \\
\hline Platypus & Ornithorhynchus anatinus & March 2007 ornAna1 \\
\hline Xenopus tropicalis & Xenopus tropicalis & August 2005 xenTro2 \\
\hline Zebrafish & Danio rerio & July 2007 danRer5 \\
\hline
\end{tabular}




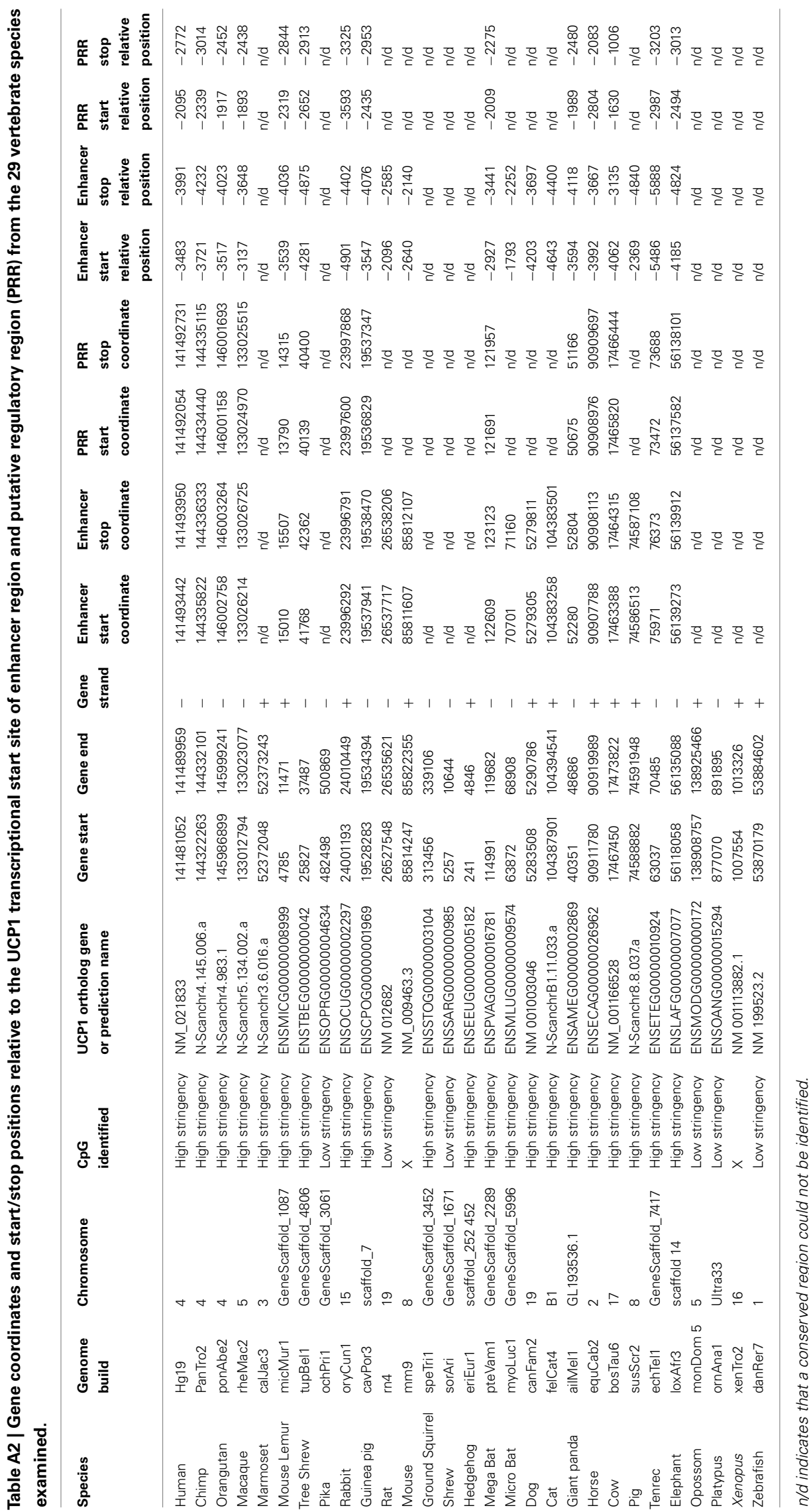


Table A3 | Primer sequences for QPCR quantification of mRNA and methylation sensitive restriction digests, bisulfite specific PCR, and pyrosequencing.

\begin{tabular}{|c|c|c|c|}
\hline Primer name & Primer sequence $\left(5^{\prime}-3^{\prime}\right)$ & PCR annealing temp ( $\left.{ }^{\circ} \mathrm{C}\right)$ & CpG positions \\
\hline Bov UCP1F & CACTAGGGAAGGACCGTCAG & 55 & \\
\hline Hom UCP1 F & TGCCCAACTGTGCAATGAA & 56 & \\
\hline Hom UCP1 R & TCGCAAGAAGGAAGGTACCAA & & \\
\hline $18 \mathrm{~S}$ & GTAACCCGTTGAACCCCATT & 56 & \\
\hline \multicolumn{4}{|c|}{ QPCR QUANTIFICATION OF METHYLATION SENSITIVE RESTRICTION DIGESTS } \\
\hline Bov Long F & GCATCGAGGGTAGAGCGTAG & 56 & \\
\hline Bov Long R & GTGTCCCACCATCCTGACTC & 56 & \\
\hline Bov Short F & TCCGGCGATATAAGTCATCC & 56 & \\
\hline Bov Short R & СTCTCCGACTTCTGCCCAGT & 56 & \\
\hline Hom $L$ and $S F$ & CCAAAGGGTGACAGAAGGTG & 56 & \\
\hline Bov Pyro F & GGAGGTAGGTAGGGGGTTGT & 56 & $1,2,3,4,5,6$ \\
\hline Bov Pyro R & BIO-AAAACCTACCCCCCAAAACAC & 56 & $1,2,3,4,5,6$ \\
\hline Bov Pyro F & GGGGATTAGGGTTTTAAGTTTTAAAGGT & 52 & $7,8,9,10$ \\
\hline Bov Pyro R & BIO-СССССАССТАССАССТАAА & 52 & $7,8,9,10$ \\
\hline Bov Pyro F & GTGGTGTTTAGTGGGAAGGTGATTATG & 52 & 11 and 12 \\
\hline Bov Pyro R & BIO-ACСТTTAAAACTAAAАСССТАAТССС & 52 & 11 and 12 \\
\hline Mouse Pyro F & GATGTTTTTGTGGTTTGAGTGTA & 58 & $1,2,3,4$ \\
\hline Mouse Pyro R & BIO-TCCCCAAAAAATCTAATTTCTAC & 58 & $1,2,3,4$ \\
\hline Mouse Pyro F & TTTTGGGGGTAGTAAGGTTAAT & 53.3 & 5 and 6 \\
\hline Mouse Pyro R & BIO-TATTACCCAACAAAAACTTTCC & 53.3 & 5 and 6 \\
\hline \multicolumn{4}{|c|}{ PYROSEQUENCING PRIMERS } \\
\hline Mouse Pyro 5-6 & TTTTTTGTTTTGAGTTGATA & & 5 and 6 \\
\hline
\end{tabular}

BIO indicates biotinylation and $C p G$ position represents $C p G$ dinucleotides successfully pyrosequenced in the bovine (Bov) and human (Hom) proximal promoters. 


Row Chr8
Cow Chr17

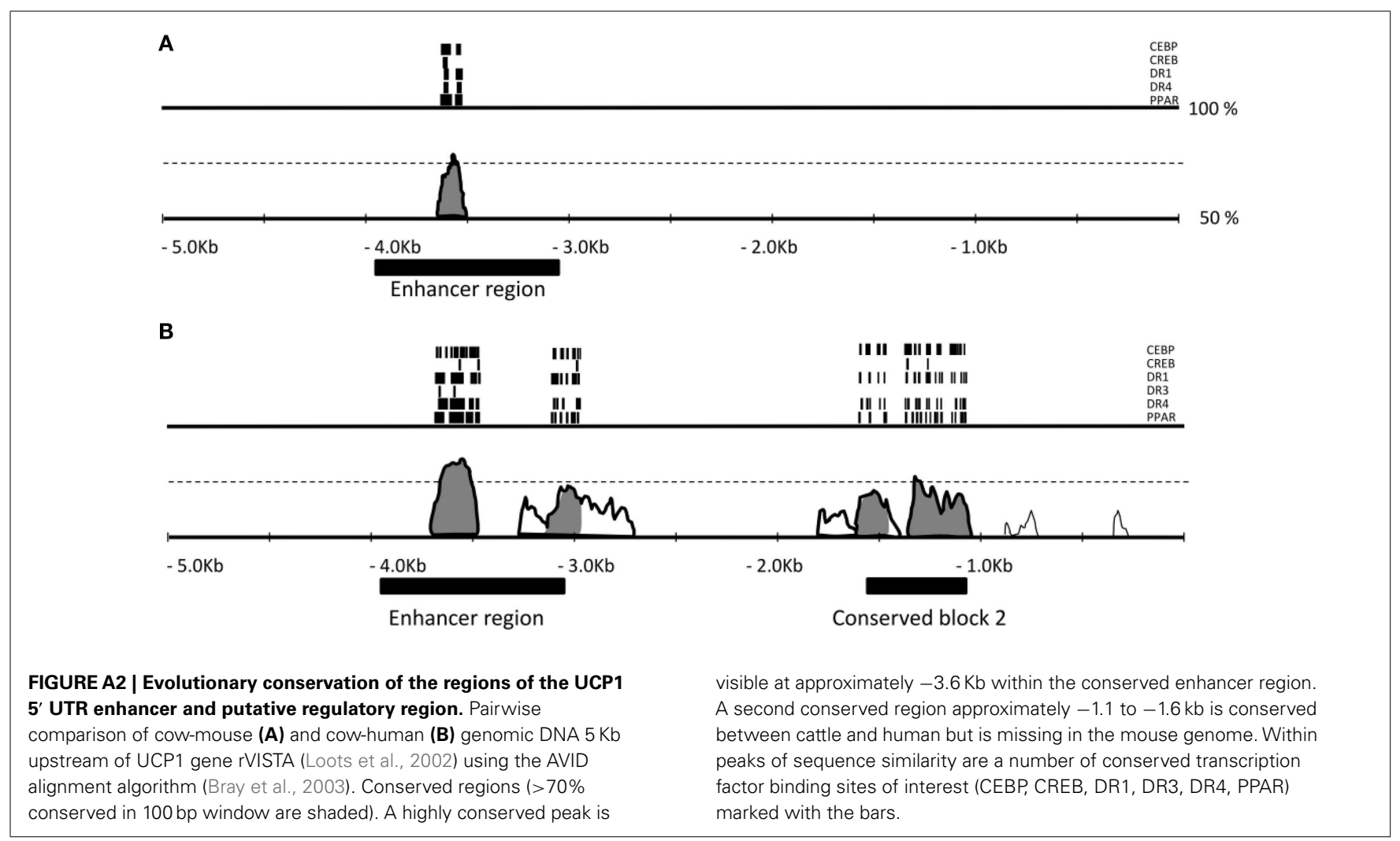




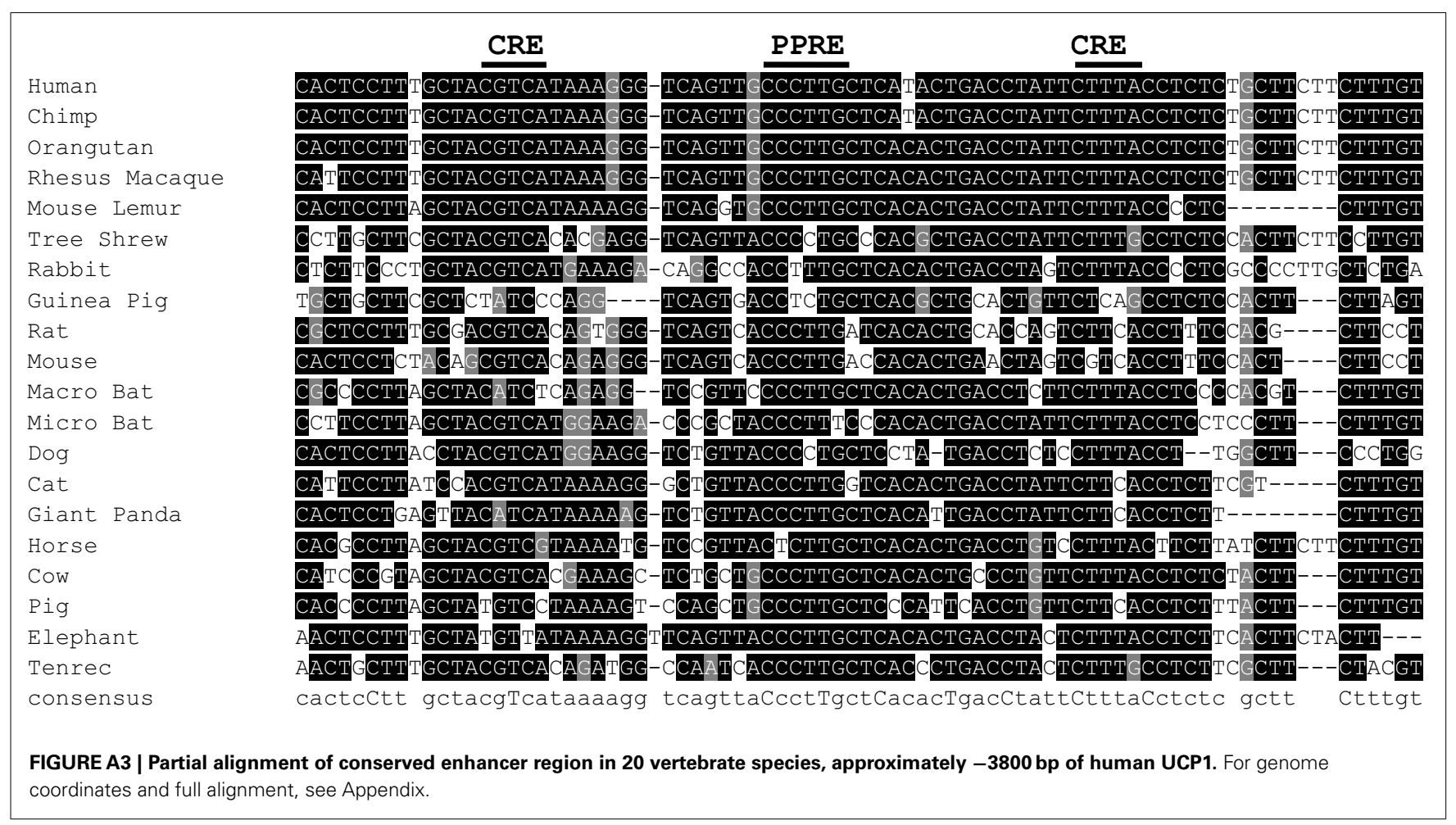




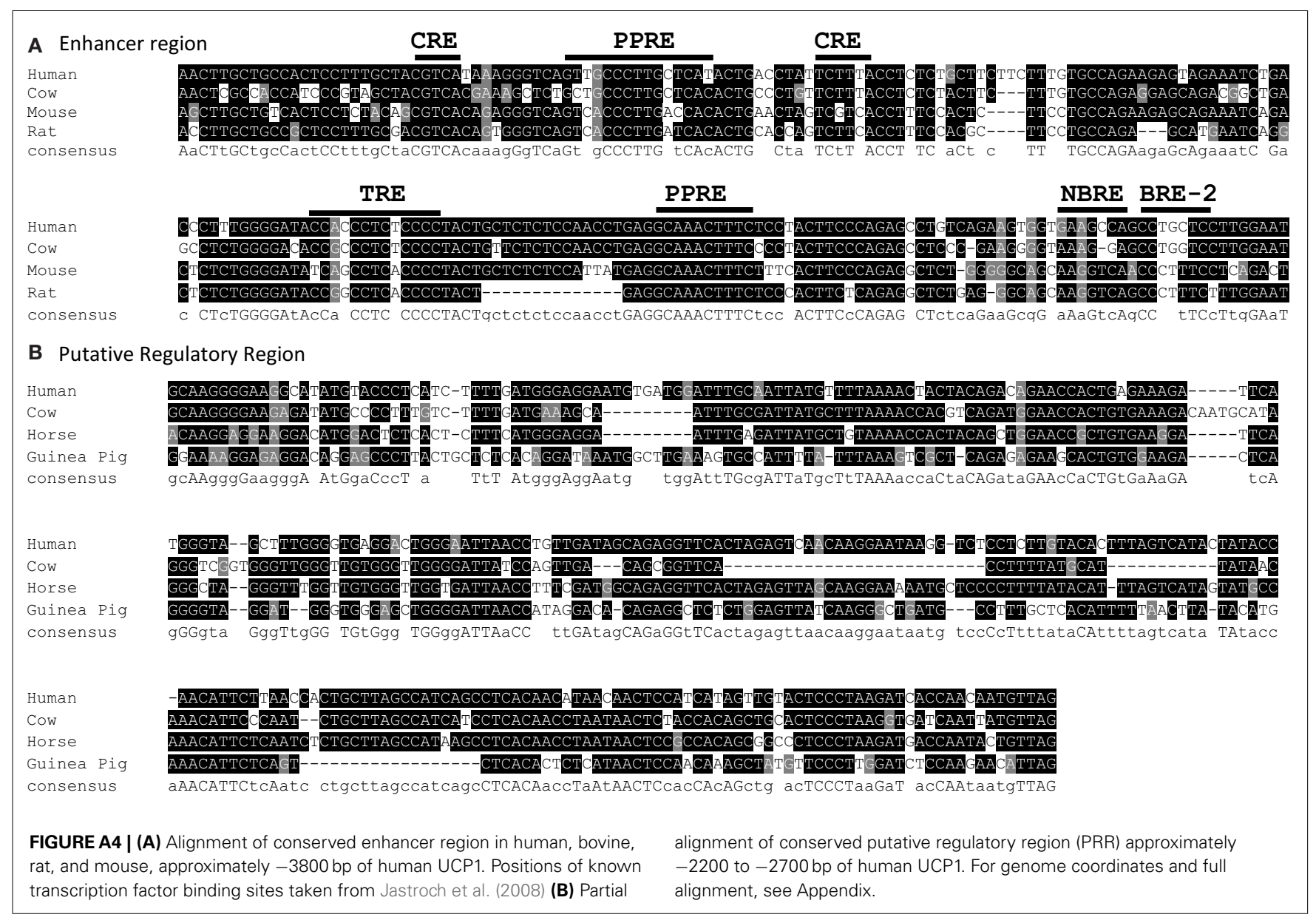


$>$ Human

ttataatctggtctcagaagccacatgg----catcagttctgtattattctattggtca aaacattcataagcctg-ccagatgcaaggggaaggcatatgta--ccctcatc-ttttg atgggaggaatgtgatggatttgcaattatgttttaaaactactac----------aGAC AGAACCACTGAGAAAG---ATTCATGGG---TA-GCTTTGGGGTGAGGACT-GGGAATTA ACCTGTTG---ATAG----CAG-AGGTTCACTAGAGTCAACAAGGAATAAGGT-CTCCTC TTGTACACTTTAGTCATACTA-TACCAACATTCTTAAC---CACTGCTTAGCCATCAGCC TCACAACATAACAACTCCATCATAGTTGTACTCCCTAAGATCACCAACAATGTTAGAGTC AAATCCGGTAGGTTTT------------TCTTTGTTTTTGTCCTCCT--GACATTTTTT CTAAACTTGAC------ACTGGTC---AGACCCAATCTTTCTTT-AATCATATTCTTAAA TACCA--GTTCTATCACTGGATATGTT-----------------------------

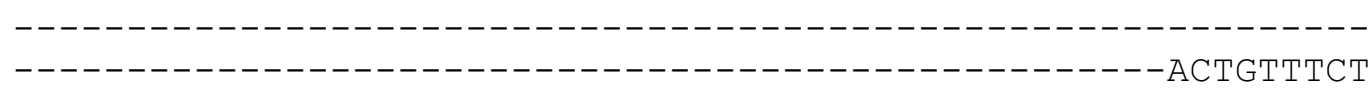

TGTTCTCACTCTACCTTTGACAAAGCCATTCTTTCCAGACTATAACTCTGGGTCTGGGTC CCCCTATGGTTTGGCCCTTGAATTCTTTTCCTAGTCCTATTTGACTAGCCCCATTTTCCC GTGAAAAGCATGCCCCTTTCATTGCATCCATATCATGACTACCAAATA

$>$ Chimp

ttataatctggtctcagaagccacatgg----catcagttctgtattattctattggtca aagcattcataagcctg-ccagatgcaaggggaaggcatatgta--ccctcatc-ttttg atgggaggaatgtgatggatttgcaattatgttttaaaGCTACTAC----------AGAC AGAACCACTGAGAAAG---ATTCATGGG---TA-GCTTTGGGGTGAGGACT-GGGAATTA ACCTGTTG---ATAG----CAG-AGGTTCACTAGAGTCAACAAGGAATAAGGT-CTCCTC TTGTACACTTTAGTCATACTA-TACCAACATTCTTAAC---CACTGCTTAGCCATCAGCC TCACAACATAACAACTCCATCATAGTTGTACTCCCTAAGATCACCAACAATGTTAGAGTC AAATCCGGTAGGTTTT-----------TCTTTGTTTTTGTCCTCCT--GACATTTTTT CTAAACTTGAC-----ACTTC----AGACCCAATCTTTCTTT-AATCATATTCTTAAA TACCA--GTTCTATCACTGGATATGTT------------------------------

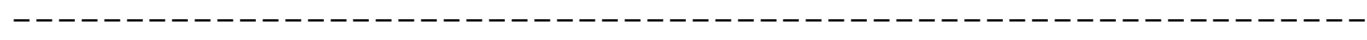

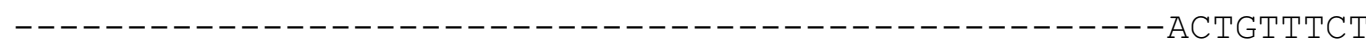
TGTTCTCACTCTACCTTTGACAAAGCCATTCTTTCCAGACTATAACTCTGGGTCTGGGTC CCCCTATGGTTTGGCCCTTGAACTCTTTTCCTAGTCCTATTTGACTAGCCCCATTTTCCC GTGAAAAGCATGCCCCTTTCATTGCATCCATATCATGACTACCAAATA

>orangutan

ttataatctggtctcagaagccacatgg----catcagttctgtattattctattggtca aagcattcataagcctg-ccagat--------------atgga--tcctcatc-ttttg atgggaggaatgtgatggatttgcaattatgttttaaaaccactac----------aGAT

\section{FIGURE A5 | Continued}


AGAACCACTGAGAAAG---ATTCATGAG---TA-GCTTTGGGGTGAGGACT-GGGAATTA ACCTGTTG---ATAG----CAG-AGGTTCACTTGAGTCAGCAAGAAATAAGGT-CTCGTC TTGTACACTTTAGTGATACTA-TACCAACATTCTTAAC---CACTGCTTAGCCATCAGCC TCACAATCTAACAACTCCATGATAGTTGTACTCCCTAAGATCACCAACAATGTTAGAGTC AAATCCGGTAGGTTTT-----------TCTTTGTTTTTGTCCTCTT--GACATTTTTT CTAAACTTGAC------ACTGGTC---ACACCCAGTCTTTCTTT-AATCATATTCTTAAA TACCA--GTTCTATCACTGGATATGTT------------------------------

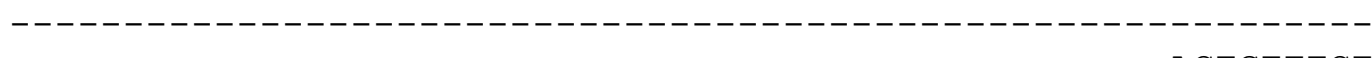

TGTTCTCACTCTACCTTMGACAAAGCCATTCTTTCCAGACT-1-0---

-----------------------------------------------------------

------------------------------------------------

$>$ Rhesus Macaque

ttataat̄̄tggtctcagaagccacatggcatccatcagttctgtattattctattggtca aagcattcataagcctg-ccagatgcaaggggaaggcacatgga--ccctcatc-ttttg atggga-gaatgtgatggatttgcaattatgttttaaaaCTACTAC----------AGAT AGAACCACTGAGAAAG---ATTCATGGG---TA-GCTTTGGGGTGAGGATT-GGGAATGA ATCCGTTG---AT-G----CAG-AGTTTCACTAGAGTCAGCAAGGAATAAGGC-CTCCTC ACGGACATTTTAGTCATACTC-TACCAACATTCTTAGC---CACTGCTTAGCCatcagcC gcacaacctaacaactccatcatagttgtactccctaagatcaccaacaatcttagagtc aaatccagtaggtttt------------tcttggtttttgtcctctt--gacatttttt ctaaacttgac------actggtca--agacccaatctttattt-aatcatgttcttaaa tacta--gctctatcactggata----------------------------------

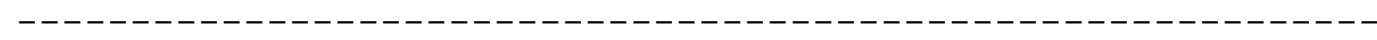
---------------------------------------------------tcact tgttctcactctacctttgacaaggccattctttccagact-------------------

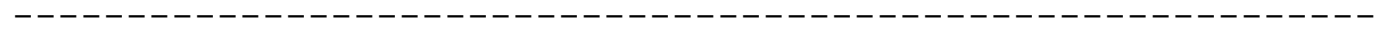

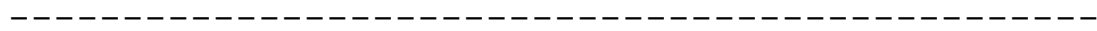

$>$ Mouse_Lemur

TTATGATTCCAGTCTCAGAAGCCACATAG----CATCAGTTC----TTATTCTATTGGTCC AAGCATTCACAAGCCTG-CCCGATGCAAGGGGAAGGCACATGGA--CCCTCA-C-TTTTG ATAAGAGTAATGTCATGTACTTGCAATTATGTTTTAAAACCACTAC---------AGAT AGAATCATGGAGAGAG---ATTCATGAG---TG-GAGCT-GAGTGAGGGTT-GGGGATTG ACCAGTTG---CTAG----CGG-AGGTTCACTAGAGTTAGCAAGGAATAATGC-CCCCTC TTATGCATTTTAGTCATCCTACTTTGGGCACGCTCAAC---CTCTGCTTGGCCGTCAGCC TCACAACTTAATAACTCCACCATAGCTGCACTGTCTAAGATCACCAATACTATCAGAGCC AAATTCAATAGGCTTT------------TC------TTTGTCCTCTT--GACATTTCTT CTAAACTTGAC-----ACTGGTTA--AGACTCAACATTTCTTT-AATCATATTCTTAAA 


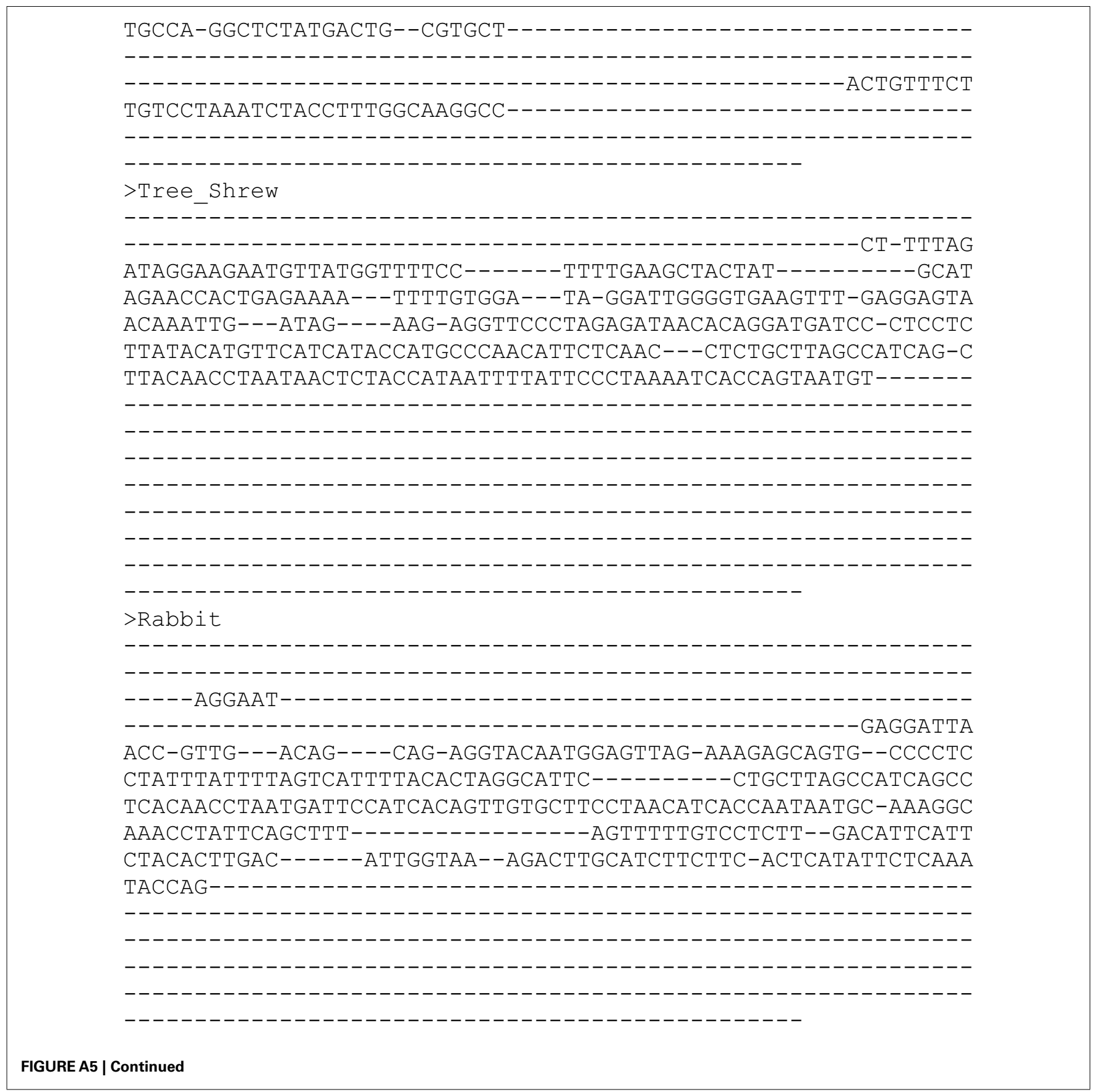


$>$ Guinea_Pig

-----------------cacatag----catccgctctgtcttacacaggcagcca cagcatcctcaggcctg-tcaggtggaaaaggagaggacaggag--cccttactgctctc acaggataaatgGCTTGAAAGTGCCATTTTA-TTTAAAGTCGCTC-----------AGAG AGAAGCACTGTGGAAG---ACTCAGGGG---TA-GGAT--GGGTGGGAGCT-GGGGATTA ACC-------ATAGGACACAG-AGGCTCTCTGGAGTTATCAAGGGCTGATGC-CTTTGC TCACATTTTTAACTTATAC---ATGAAACATTCTCAGT-------------------C TCACACTCTCATAACTCCAACAAAGCTATGTTCCCTTGGATCTCCAAGAACATTAGACCC AAATCCAGTAGCCTGTGTCTGTCTGTCGGTCTATCTCTTTGTCACCTCCTGACATTTATT GCAAACTTGAC-----ACTGA-----AGCCCCAGCCCTTCTTT-CAATATAGTCTCAAA TACCA-AGTTCTAAGACCCTACATGCT-----------------------------

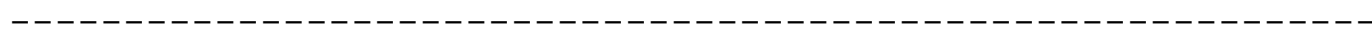
--------------------------------------------------ACTGTTTCT TGTCCTAACTCTACCTGTGGCCA-GTCATTCTTTCCAGACC-----------------

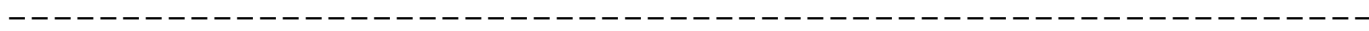
$>$ Macro Bat TTATGATCCAGCCTCGGAAACTACATAG----CATCAGCTCCGTCTTATCCTATTGGTCA AAGCGTTTACAAGCCGGCCCAGAAGTGAGGGGGAGGAACATGGA--CCCTCACC-TTTTG ATGGGAGAGACT---------TGGAATTATGCTTTAAAACTACTAC----------AGAT GGAAGCACCCATGAAGG-GGTTCAGGGG---TAGGGGTTGGGTTGTGAGTTGGGGGATTA GCCAGCCAGCCATAG----CAGAAAGCTCACTAGCGTTGGCAAGAAATGAT--------

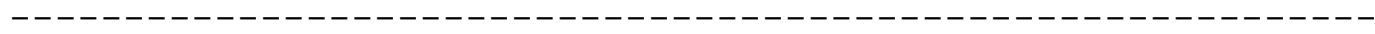

------------------------------------------------------GACCCTTCTT

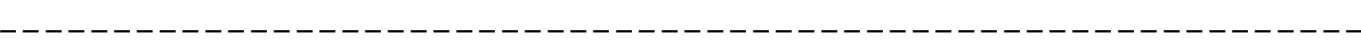

----------------------------------------------------------

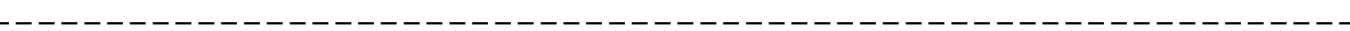

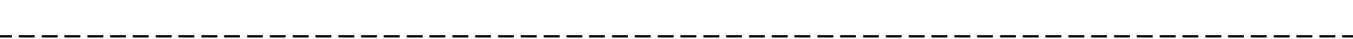

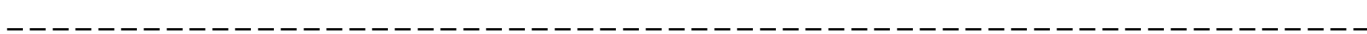

----------------------------------------------1

$>$ Giant_Panda

tcatgā̄ctagcctcagaaaccacatag----catcagttctgtcttaatctgttggtta aagcattcacaagcctgcccagatgcaaggggaagggacatgga--ccctcacc-cttta acagga-gaat---------gtgtGATTATGCTTTAAAACAACTAC----------AGGT GGAA-CATTGTGAACG---ATTCAGG-T---TA-GGGTCGGGTTGTGGGTT-GAGGACTA ACCAGTTG---ATAG----C---TAACTCTCTAGAGTTAGCTAGGAAAAATGC-CCCCCC 
CTTTACA-TCTAGTCACACTATACCCAACATTCTCAAT---CTCTGCTTGGCCATCAGCC TCAC--CCTAATGACTGCACCACA-CTGCACTCCCTAAGATGACCAATAGTGTTAGAGCC AAACCCTACAGGCTTT-----------TCTTCATTTCTGTCCTCTTTCGACATTTCTT CTAAACTTCAT-----GCTAGTCA--AGACCCCACCCTTCCTTAAATTATATTCTCAAG TACCATGGTTCCATGACCGGATACACT

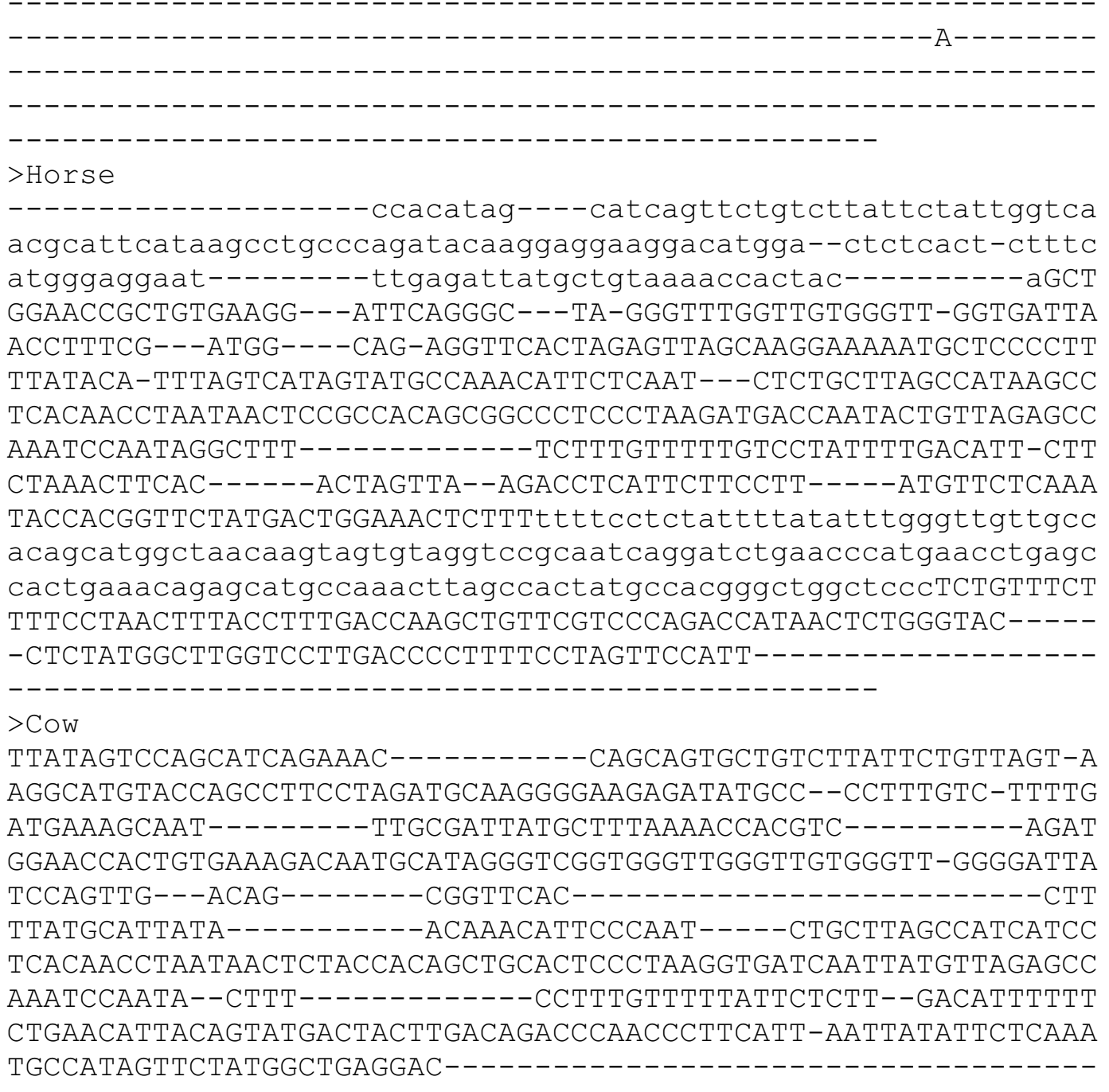




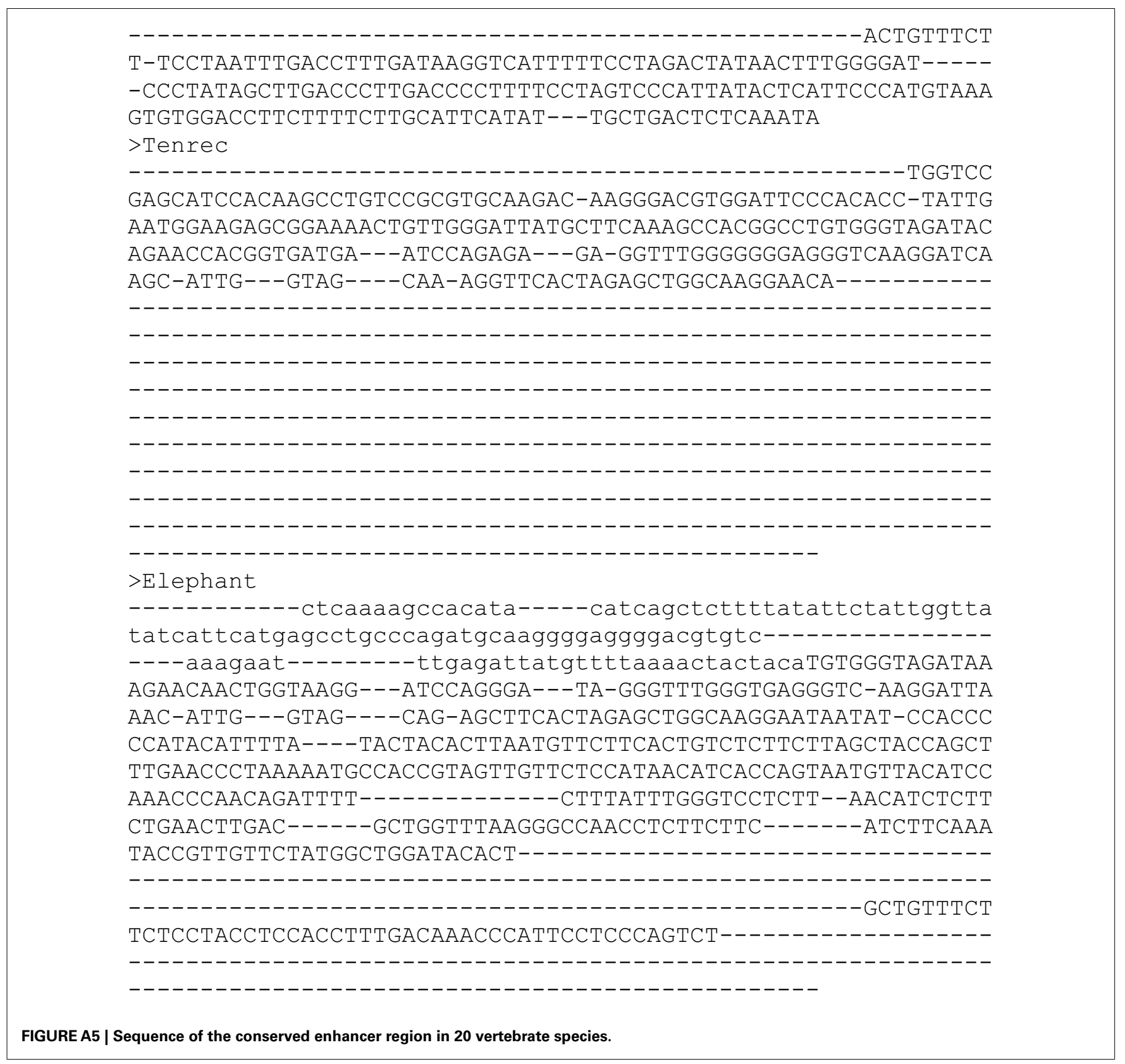

